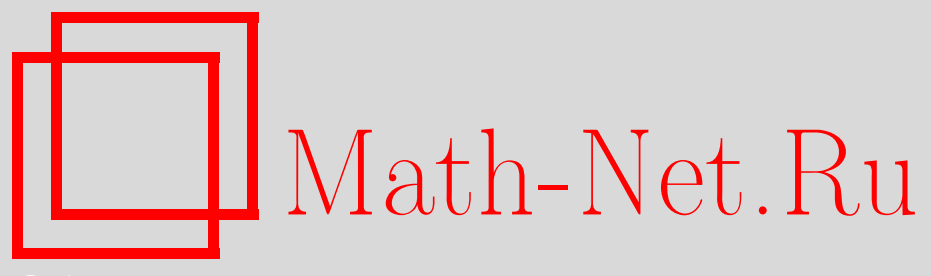

М. Д. Абловиц, Б. Илан, Э. Шонбрун, Р. Пьестун, Двумерные солитоны в нерегулярных решеточных системaх, TMФ, 2007, том 151, номер 3, 345-359

DOI: https://doi.org/10.4213/tmf6050

Использование Общероссийского математического портала Math-Net.Ru подразумевает, что вы прочитали и согласны с пользовательским соглашением http://www . mathnet.ru/rus/agreement

Параметры загрузки:

IP : 54.198 .55 .26

26 апреля 2023 г., 14:03:07

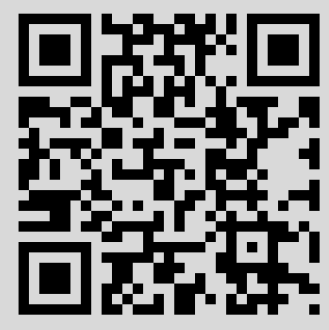




\title{
ДВУМЕРНЫЕ СОЛИТОНЫ В НЕРЕГУЛЯРНЫХ РЕШЕТОЧНЫХ СИСТЕМАХ
}

\begin{abstract}
Локализованные нелинейные моды (солитоны) рассчитаны и исследованы в случае полубесконечной щели спектра фокусирующего двумерного нелинейного уравнения Шредингера с различными нерегулярными потенциалами решеточного типа. Потенциалы характеризуются такими существенными отклонениями от периодичности, как дефекты вакансий, краевые дислокации, а также квазикристаллическая структура. Расчет солитонов ведется на основании спектральной вычислительной схемы с неподвижной точкой. Зависимость мощности солитона от собственных значений определяет области параметров самофокусирующейся неустойчивости; эти результаты сравниваются с результатами прямого численного решения нелинейного уравнения Шредингера. Показано, что в общем случае солитоны с центром вблизи локального решеточного максимума претерпевают коллапс. Более того, квазикристаллические солитоны $N$-го порядка приближаются в пределе больших $N$ к бесщелевым солитонам.
\end{abstract}

Ключевые слова: солитоны, локализованные моды на решетках, нелинейная оптика, самофокусировка луча, квазикристаллы.

\section{1. ВВЕДЕНИЕ}

Солитоны представляют собой локализованные нелинейные волны и встречаются в различных областях физики. Знание их свойств дает глубокое и фундаментальное понимание сложных нелинейных систем. В последнее время наблюдается значительный интерес к изучению солитонов, образующихся в системах с периодическими потенциалами, или решетками, в частности, таких, которые можно создать в нелинейных оптических материалах [1]-[5]. В периодических решетках солитоны могут образовываться, если константа распространения, или собственное значение,

${ }^{*}$ Department of Applied Mathematics, University of Colorado, Boulder, Colorado 80309-0526, USA

${ }^{\dagger}$ School of Natural Sciences, University of California, Merced, P.O. Box 2039, Merced, California 95344, USA. E-mail: bilan@ucmerced.edu

${ }^{\ddagger}$ Department of Electrical and Computer Engineering, University of Colorado, Boulder, Colorado 80309-0425, USA 
лежит внутри неких областей, часто называемых щелями; эта концепция, заимствована из теории линейного распространения Флоке-Блоха. Однако в сложных системах внешний потенциал может иметь гораздо более общий вид и с физической точки зрения может быть более содержательным, чем периодическая решетка. Например, атомные кристаллы могут иметь различные нерегулярности, такие как дефекты и краевые дислокации, а также квазикристаллические структуры, у которых есть дальний ориентационный порядок, но нет трансляционной симметрии [6]-[8]. Вообще говоря, если периодичность решетки слегка возмущена, то зонно-щелевая структура и солитонные свойства также слегка возмущаются, но во всем остальном ожидается, что солитоны проявляют себя в целом таким же образом, как и в идеально периодическом случае [9], [10]. Однако существование и свойства многомерных солитонов в случае, когда внешний потенциал сильно отклоняется от периодического, остаются пока в большой степени неисследованными.

В настоящей работе мы ведем поиск двумерных (2D) солитонов в решетках, обладающих дефектами вакансий, краевыми дислокациями и квазикристаллическими структурами. Мы используем спектральный метод с неподвижной точкой для вычисления основных состояний фундаментального нелинейного уравнения Шредингера (НУШ). Солитоны на нерегулярных решетках вычисляются аналогично солитонам на периодической решетке. Сравнительное исследование зависимости мощности солитонов от их собственных значений приводит к важным результатам, касающимся мощности солитонов, краев щели и свойств устойчивости. Эволюция солитонов исследуется путем прямого численного моделирования, показывающего, что слегка возмущенные одиночные волны в нерегулярных решетках могут либо испытывать колебания с малыми или большими амплитудами, либо претерпевать коллапс. Заметим, что физические свойства квазикристаллических потенциалов, создаваемых оптическим способом, вызвали значительный интерес, и недавно были построены локализованные дефекты внутри оптических решеток [11], [12]. Наши результаты также могут быть применены к фотонным зонно-щелевым системам, для которых недавно использовались ранее неизвестные экспериментальные методы создания нерегулярных решеточных структур [12]-[15].

Мы исследуем нелинейную систему, подчиняющуюся фокусирующему $(2+1)$ мерному НУШ (в безразмерных единицах) с внешним потенциалом

$$
i u_{z}+\Delta u+|u|^{2} u-V(x, y) u=0 .
$$

В оптике $u(x, y, z)$ соответствует комплекснозначной медленно меняющейся амплитуде электрического поля в плоскости $(x, y)$, распространяющегося в направлении $z$ (которое также играет роль времени), $\Delta u \equiv u_{x x}+u_{y y}$ описывает дифракцию, кубичный член в (1) возникает из нелинейного (керровского) изменения показателя преломления, а потенциал $V(x, y)$ соответствует модуляции линейного показателя преломления среды. Уравнение (1) также описывает динамику некоторых бозе-эйнштейновских конденсатов, где $u(x, y, z)$ - волновая функция среднеполевого атомного конденсата, удерживаемого в потенциале [16]. 
Мы ищем локализованные решения уравнения (1) в виде $u(x, y, z)=f(x, y) e^{-i \mu z}$, где $\mu$ - константа распространения (или собственное значение), а $f(x, y)$ - вещественнозначная локализованная функция, удовлетворяющая в соответствии с (1) нелинейному уравнению на собственные значения

$$
\Delta f+\left[\mu+|f|^{2}-V(x, y)\right] f=0 .
$$

В настоящей работе мы рассматриваем потенциалы, которые можно записать в виде интенсивности суммы $N$ фазово-модулированных плоских волн, т.е. как

$$
V(x, y)=\frac{V_{0}}{N^{2}}\left|\sum_{n=0}^{N-1} e^{i \overrightarrow{\mathbf{k}}_{n} \cdot \overrightarrow{\mathbf{x}}+i \theta_{n}(x, y)}\right|^{2},
$$

где $V_{0}>0$ - константа, $\overrightarrow{\mathbf{x}}=(x, y), \overrightarrow{\mathbf{k}}_{n}$ - волновой вектор, $\theta_{n}(x, y)$ - фазовая функция, с помощью которой вводятся нерегулярности, а $N^{2}$ в нормировке приводит к тому, что $V_{0}$ - максимальная глубина потенциала, т.е. $V_{0}=\max _{x, y} V(x, y)$. Такие 2Dпотенциалы можно физически реализовать в оптике путем интерференции плоских волн и фазовых функций [12]. Например, эти фазовые функции можно составить из различных конфигураций вихрей [17], которые, в свою очередь, можно создать, используя порожденные компьютером голограммы [12].

Для расчета солитонных решений уравнения (2) мы используем спектральный вычислительный метод с неподвижной точкой [18], см. приложение.

\section{2. ЗОННО-ЩЕЛЕВАЯ СТРУКТУРА}

Хорошо известно, что если уравнение (2) линейно, а потенциал периодический, T.e.

$$
\Delta f+[\mu-V(\overrightarrow{\mathbf{x}})] f=0, \quad V(\overrightarrow{\mathbf{x}})=V(\overrightarrow{\mathbf{x}}+\overrightarrow{\mathbf{L}}),
$$

то согласно теории Флоке-Блоха можно записать решение в виде

$$
f(\overrightarrow{\mathbf{x}})=e^{i \overrightarrow{\mathbf{k}} \cdot \overrightarrow{\mathbf{x}}} p(\overrightarrow{\mathbf{x}}),
$$

где $p(\overrightarrow{\mathbf{x}})=p(\overrightarrow{\mathbf{x}}+\overrightarrow{\mathbf{L}})$ - ограниченная функция с той же периодичностью, что и $V(x, y)$, a $\overrightarrow{\mathbf{k}}(\mu)=\left(k_{1}, k_{2}\right)$ может принадлежать одному из следующих трех классов (ср. [19]):

- $\overrightarrow{\mathbf{k}}(\mu)$ принимает вещественные значения, и в этом случае функция $f(\overrightarrow{\mathbf{x}})$ ограничена, а о $\mu$ говорят, что оно лежит в зоне (или на спектре).

- $\overrightarrow{\mathbf{k}}(\mu)$ строго комплексна, $f(\overrightarrow{\mathbf{x}})$ неограничена, а $\mu$ находится в щели.

- $e^{i \overrightarrow{\mathbf{k}} \cdot \overrightarrow{\mathbf{L}}}= \pm 1, f(\overrightarrow{\mathbf{x}})$ является (полу)периодической, что в общем случае имеет место при счетно-бесконечной последовательности собственных значений $\mu_{1}<\mu_{2}<\cdots$ с предельной точкой на бесконечности.

В настоящей работе мы интересуемся локализованными нелинейными, а не линейными состояниями. Более того, потенциал является непериодическим. Однако мы полагаем, что понятие зонно-щелевых состояний полезно и в этих случаях. В нашем исследовании мы провели компьютерный расчет нелинейных локализованных состояний в полубесконечной щели $-\infty<\mu<\mu_{\max }$, где $\mu_{\max }$ есть край щели, равный максимальной величине, для которой вычислительный метод дает сходимость к локализованной моде. 

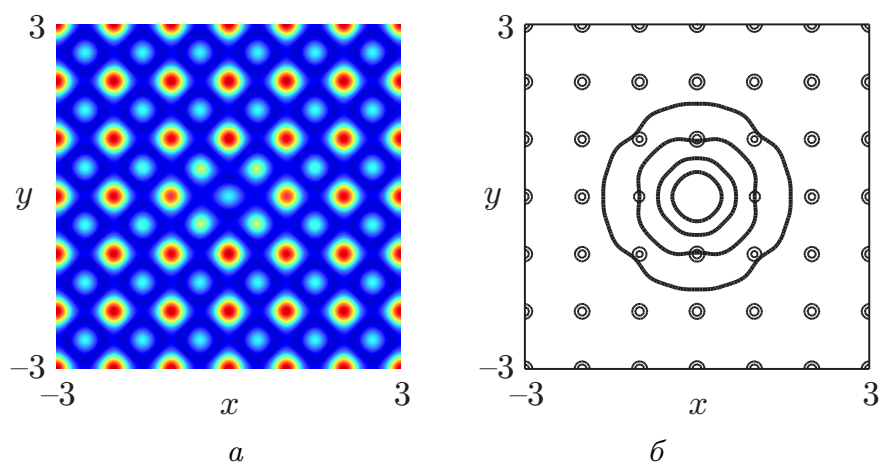

Рис. 1. Контурное представление решетки с дефектом вакансии, т.е. потенциал (4) при $V_{0}=12.5$ и $K=k_{x}=k_{y}=2 \pi$. Темные кружки соответствуют локальным максимумам (а). Контурное изображение солитона при $\mu=0.5$, наложенное на решетку (б). Для наглядности представлена только малая часть вычислительной области $[-10,10]^{2}$.

\section{3. ДЕФЕКТ ВАКАНСИИ}

Первый тип потенциала (3), который мы рассмотрим, представляет собой нерегулярную 2D-квадратную решетку с дефектом вакансии (рис. 1a),

$$
V(x, y)=\frac{V_{0}}{25}\left|2 \cos \left(k_{x} x\right)+2 \cos \left(k_{y} y\right)+e^{i \theta(x, y)}\right|^{2},
$$

где $k_{x}=k_{y}=K$, а фазовая функция $\theta(x, y)$ имеет вид

$$
\theta(x, y)=\operatorname{arctg}\left(\frac{y-y_{0}}{x}\right)-\operatorname{arctg}\left(\frac{y+y_{0}}{x}\right)
$$

С физической точки зрения $\theta(x, y)$ соответствует двум фазовым дислокациям первого порядка, смещенным друг относительно друга в направлении $y$ на расстояние $2 y_{0}$. Дефект вакансии можно получить, если использовать $y_{0}=\pi / K$. Заметим, что "вакансия" в начале координат порождается непрерывной функцией, а вдали от начала координат потенциал (4) локально является квадратной решеткой с периодом $2 \pi / K$. Используя описанный в приложении вычислительный метод, находим локализованные моды (солитоны) уравнения (2) с потенциалом (4) с центром вблизи вакансии, как показано на рис. 1б. В некоторых отношениях они напоминают солитоны с центром вблизи минимума периодической квадратной решетки. В ходе дальнейших исследований выясняется, что если центр солитона смещается дальше от вакансии, то его профиль и зонно-щелевая структура сходятся к аналогичным характеристикам для соответствующей периодической решетки (т.е. мы получаем уравнение (4) при $\theta(x, y) \equiv 0)$. 


\section{4. КРАЕВАЯ ДИСЛОКАЦИЯ}

Подобным образом из уравнения (3) при

$$
V(x, y)=\frac{V_{0}}{25}\left\{2 \cos \left[k_{x} x+\theta(x, y)\right]+2 \cos \left(k_{y} y\right)+1\right\}^{2},
$$

где $k_{x}=k_{y}=K$, а функция фазовой дислокации имеет вид $\theta(x, y)=3 \pi / 2-$ $\operatorname{arctg}(y / x)$, получаем решетку с краевой дислокацией, аналогичную тем, что можно обнаружить в атомных кристаллах [8], [12]. Из рис. 2а видно, что такая дислокация не похожа на точечный дефект, коль скоро плотность узлов решетки изменяется в вертикальном направлении вдоль решетки. Несмотря на эту сильную нерегулярность оказывается, что солитоны существуют в окрестности фазовой дислокации. Из рис. $2 б$ видно, что солитон имеет асимметричную форму. Центр солитона находится ниже фазовой дислокации между соседними локальными максимумами решетки. В этом отношении он похож на солитон с центром вблизи минимума решетки. Следует отметить, что в ходе расчетов начальная точка была расположена вблизи начала координат, а в процессе итераций решение смещалось вверх вдоль оси $y$, до тех пор, пока не была достигнута сходимость.

Неожиданное наблюдение, которое можно сделать на основании приведенного далее рис. 7 , состоит в том, что край щели при краевой дислокации появляется при $0.9<\mu_{\max }<0.95$, т.е. при величине значительно меньшей, чем для других решеток. В действительности во время вычислений при возрастании $\mu$ возникает интересное явление. При $\mu=0.9$ достигается надежная сходимость (величина $\delta=10^{-10}$, определение относительной погрешности $\delta$ см. в приложении), и в этом случае решение лежит между локальными максимумами, показанными на рис. За. При $\mu=0.95$ решение сначала сходится в том же месте $\left(\delta=10^{-5}\right)$. Однако такая "сходимость" обманчива, поскольку при дальнейших итерациях решение смещается вдоль оси $y$, “скользя" вверх между двумя локальными максимумами, и в конце концов сходится $\left(\delta=10^{-10}\right)$ между четырьмя локальными максимумами, образующими одну решеточную ячейку выше краевой дислокации (рис. За). Дальнейшие исследования показывают, что при $0.95<\mu<2$ солитоны остаются на одну ячейку решетки выше, чем дислокация. Эти солитоны "видят" более периодическую решетку, коль скоро ближайшие четыре решеточных максимума располагаются приблизительно симметрично и на одинаковом расстоянии, а потому эти солитоны похожи на солитоны периодической квадратной решетки. Зависимость мощности этих солитонов от их собственных значений очень похожа на соответствующую зависимость для периодической решетки (см. далее рис. 7). Таким образом, краевая дислокация очевидным образом уменьшает размер эффективной нелинейной щели.

\section{5. КВАЗИКРИСТАЛЛИЧЕСКИЕ СОЛИТОНЫ ПЕНРОУЗА}

Далее мы исследуем солитоны на квазикристаллических решетках. Такие решетки появляются естественным образом в некоторых молекулах [6], [8], они были 

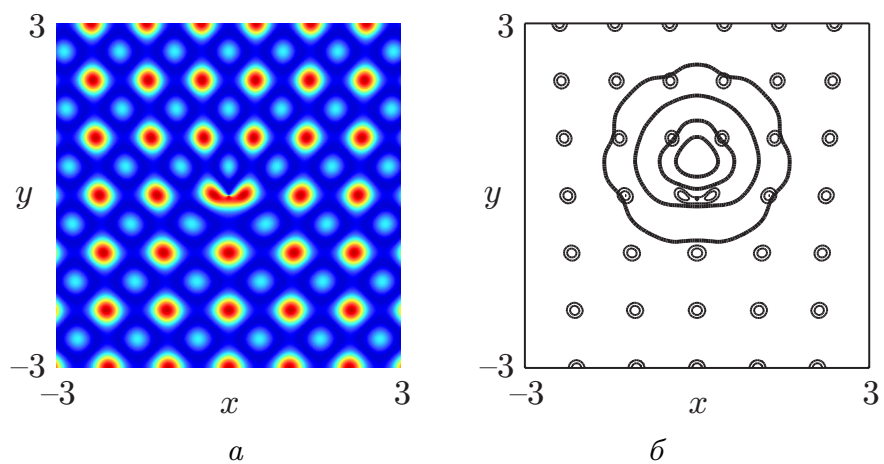

Рис. 2. То же, что на рис. 1, для решетки с краевой дислокацией (уравнение (5)); параметры решетки те же, что и на рис. $1, \mu=0.5$. Пик солитона расположен в точке $(0,0.68)$.
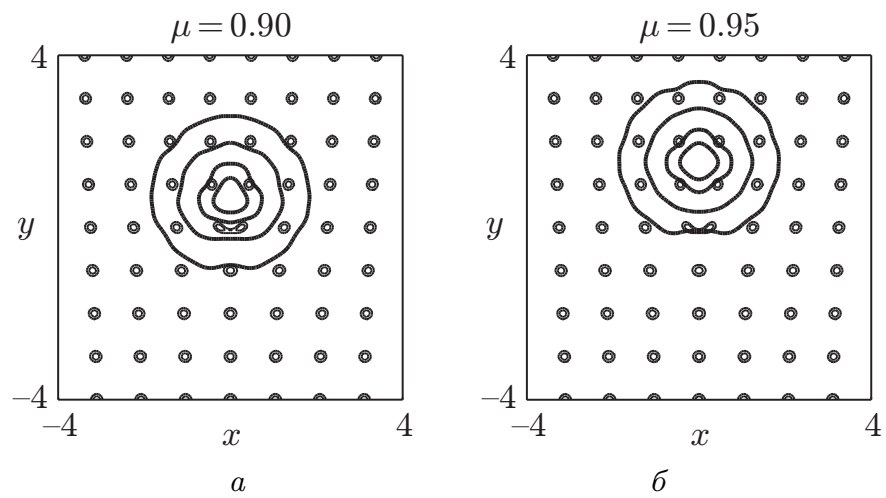

Рис. 3. Если константа распространения $\mu \geqslant 0.95$, солитон в процессе сходимости сдвигается на одну ячейку решетки вверх по оси $y$. Край эффективной нелинейной щели при краевой дислокации возникает при $0.9<\mu_{\max }<0.95$.

созданы и исследовались в оптике [20]-[22], а также при анализе бозе-эйнштейновских конденсатов [23]. Важно отметить, что Фридман с соавторами недавно предсказали и наблюдали солитоны в квазикристаллах Пенроуза, полученных с использованием метода оптической индукции [24]. В нашей работе оптический потенциал образован дифракционной картиной дальнего поля с маской точечных апертур, расположенных в $N$ вершинах правильного многоугольника. Соответствующий потенциал имеет вид

$$
V_{N}(x, y)=\frac{V_{0}}{N^{2}}\left|\sum_{n=0}^{N-1} e^{i\left(k_{x} x+k_{y} y\right)}\right|^{2},
$$

где $\left(k_{x}, k_{y}\right)=(K \cos (2 \pi n / N), K \sin (2 \pi n / N))$. Потенциал (6) при $N=2,3,4,6$ дает периодические решетки, которые соответствуют стандартным 2D-кристаллическим 

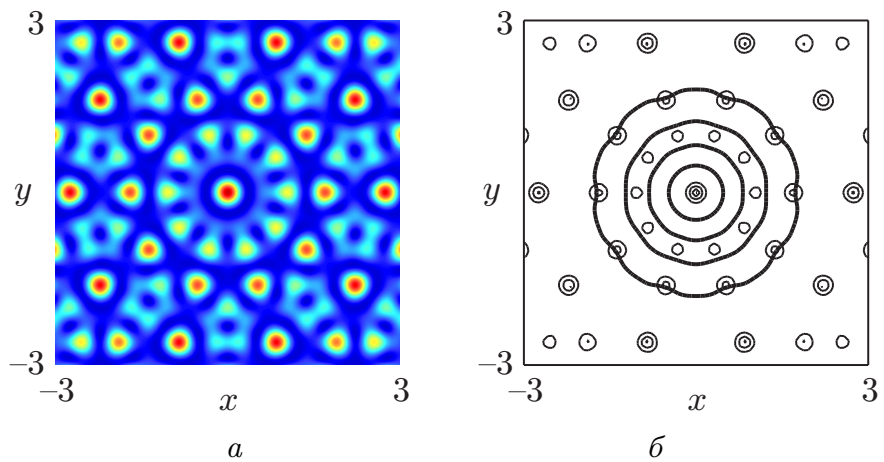

Рис. 4. То же, что на рис. 1 , для решетки Пенроуза (формула (6) при $N=5$ ) и соответствующий солитон с центром вблизи центра решетки (максимума); параметры решетки те же, что и на рис. $1, \mu=0.5$.

структурам. Все другие значения $N$ соответствуют квазикристаллам, которые обладают локальной симметрией вблизи начала координат и дальним порядком, но в отличие от периодических кристаллов не инвариантны относительно пространственных трансляций [7]. Ниже мы сосредоточимся на случае $N=5$ (рис. 4a); о соответствующей этому случаю решетке часто говорят как о квазикристалле Пенроуза.

Мы находим солитоны на решетке Пенроуза (рис. 4б) с центрами вблизи начала координат, которое является глобальным максимумом потенциала решетки. Подобно солитонам с центрами в максимуме периодических решеток солитоны Пенроуза имеют в центре небольшую впадину (рис. 5). Дальнейшие исследования показывают, что солитоны Пенроуза с центром вблизи локальных минимумов не имеют впадины подобно их аналогам для периодических решеток (рис. 6).

Заметим, что в некоторых предыдущих исследованиях решеток Пенроуза рассматривались различные решетки с цилиндрами из керровского материала, локализованными в вершинах (виртуального) замощения Пенроуза, окруженного воздухом [20]-[22], [25], [26]. Среда, которую мы здесь рассматриваем, является однородной и характеризуется постоянным коэффициентом Керра и непрерывной модуляцией линейного показателя преломления (6).

\section{6. СООТНОШЕНИЕ МЕЖДУ МОЩНОСТЬЮ СОЛИТОНА И СОБСТВЕННЫМИ ЗНАЧЕНИЯМИ}

Для сравнения различных решеточных солитонов с центрами вблизи локальных минимумов или локальных максимумов мы приводим на рис. 7 зависимость мощности солитона $P=\int f^{2}(x, y) d x d y$ от собственного значения $\mu$ как для всех рассмотренных выше решеток, так и для соответствующей периодической квадратной решетки (см. формулу (4) при $\theta(x, y) \equiv 0)$. Заметим, что все описанные выше решетки имеют одну и ту же максимальную глубину потенциала $V_{0}=12.5$, а также 

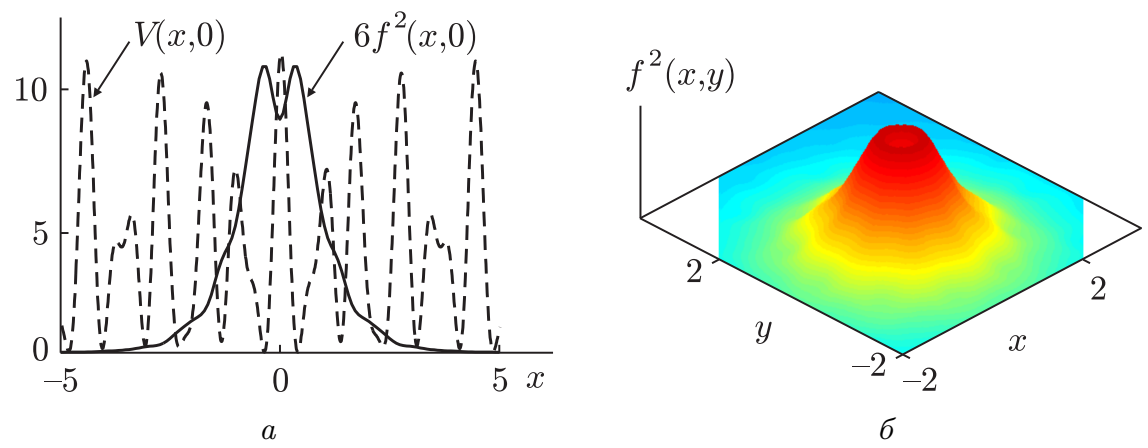

Рис. 5. Аналогично солитону с центром вблизи максимума периодической решетки солитоны Пенроуза могут иметь небольшую впадину, которая становится более выраженной при больших значениях $\mu$, т.е. вблизи края щели. Сечение $6 f^{2}(x, 0)$ вдоль оси $y=0$ для солитона Пенроуза (сплошная линия) при $\mu=2$, наложенное на потенциал $V(x, 0)$ решетки (штриховая линия) (a). Трехмерное изображение интенсивности солитона, на котором видна впадина (б).

общее значение фонового волнового числа $K=2 \pi$, при этом для решетки Пенроуза $K$ можно считать “локальным" волновым числом (см. формулу (6)).

Локализованные моды рассчитываются в полубесконечной щели $\mu<\mu_{\max }$ для некоторого зависящего от решетки значения $\mu_{\max }$. Когда собственное значение превосходит $\mu_{\max }$, численный метод обычно сходится к расширенному состоянию (однако имеются исключения, см. ниже). Кроме того, сравнение показывает следующее (см. рис. 7).

1. Для значительного интервала собственных значений мощность солитонов в случаях дефекта вакансии, краевой дислокаци и квазикристаллической решетки Пенроуза ниже, чем мощность соответствующих солитонов в случае периодической решетки. В частности, для всех исследованных в настоящей работе решеток самую низкую мощность имеют солитоны в случаях вакансий и краевых дислокаций.

2. Дефект вакансии слабо влияет на размер щели $\left(\mu_{\max } \approx 2\right)$, но значительно понижает порог мощности, т.е. минимальную мощность солитона по всей щели. Однако заслуживает внимания тот факт, что для всех этих потенциалов порог мощности положителен.

3. Краевая дислокация (штриховая линия) уменьшает размер щели $\left(0.9<\mu_{\max }<\right.$ 0.95), как объяснялось в разделе 4.

4. Солитон Пенроуза имеет несколько больший размер щели $\left(\mu_{\max } \approx 2.2\right)$ по сравнению с солитонами на периодической квадратной решетке. Однако для солитонов с центрами вблизи максимумов периодических квадратных решеток и решеток Пенроуза мощность ведет себя одинаково, что представляется несколько неожиданным результатом, поскольку структуры этих решеток сильно различаются. 


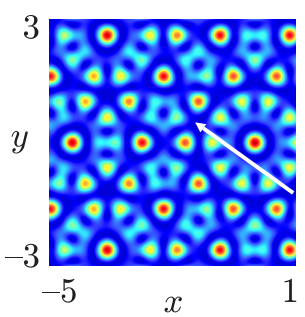

$a$

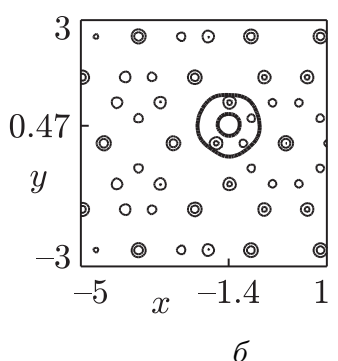

$f^{2}(x, y)$

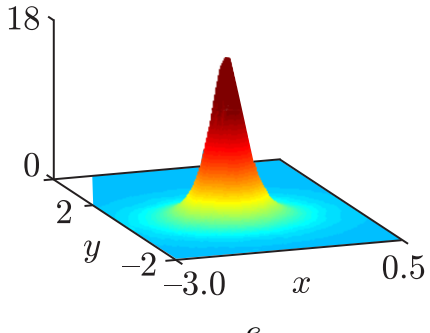

Рис. 6. Солитон с центром вблизи локального минимума в точке $(-1.4,0.47)$ (показана стрелкой) для решетки Пенроуза; использованы те же параметры решетки, что и на рис. $4, \mu=-1$ (а). Контурное представление амплитуды солитона, наложенное на фоновую решетку (б). Трехмерное изображение интенсивности солитона $f^{2}(x, y)$; видно, что профиль солитона монотонно убывает и не имеет впадины (в).

\section{7. ЭВОЛЮЦИЯ И УСТОЙчИВОСТЬ}

Вопрос эволюции солитона под действием возмущений является важным для приложений. Для его изучения мы проведем прямые вычисления на основе уравнения (1), используя различные потенциалы, при этом роль начальных условий играет солитон со случайным шумом в амплитуде и фазе, относительная величина которого составляет $1 \%$. В случае общего положения получаем следующее.

Солитоны с центрами вблизи решеточных минимумов претерпевают колебания с небольшой амплитудой при $d P / d \mu<0$ (см. рис. 8, иллюстрирующий устойчивость для решетки с дефектом вакансии) и колебания с большой амплитудой при $d P / d \mu>$ 0 (см. рис. 9, иллюстрирующий неустойчивость для решетки с краевой дислокацией).

Солитоны с центрами вблизи решеточных максимумов, как правило, претерпевают коллапс после распространения на конечное расстояние (см. рис. 10 для решетки Пенроуза).

\section{8. КВАЗИКРИСТАЛЛИЧЕСКИЕ СОЛИТОНЫ ВЫСОКОГО ПОРЯДКА}

Решетка Пенроуза представляет собой квазикристаллическую решетку пятого порядка, т.е. описывается уравнением (6) при $N=5$. Интересно исследовать солитоны более высокого порядка на квазикристаллической решетке. Мы рассмотрим случай, когда направления приходящих волн разделены равными углами, т.е.

$$
V_{N}(x, y)=\frac{V_{0}}{N^{2}}\left|\sum_{n=0}^{N-1} e^{i K\left(x \cos \theta_{n}+y \sin \theta_{n}\right)}\right|^{2},
$$

где $\theta_{n}=2 \pi n / N$. В пределе бесконечного числа волн $N \rightarrow \infty$ квазикристаллическая решетка приближается к решетке Бесселя. Это можно установить, используя 


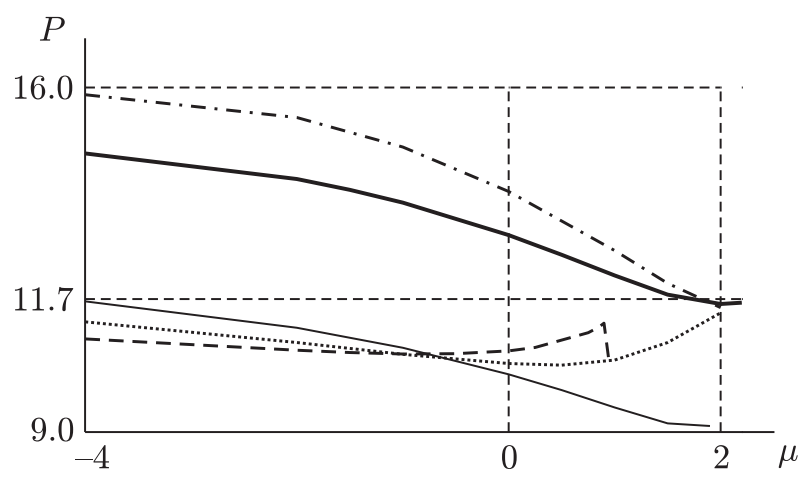

Рис. 7. Мощность солитона $P$ как функция собственного значения $\mu$ внутри полубесконечной щелевой зоны для солитонов на решетке Пенроуза (жирная сплошная линия), решетке с дефектом вакансии (тонкая сплошная линия), решетке с краевой дислокацией (штриховая линия), а также для солитонов в максимумах (штрихпунктирная линия) и минимумах (пунктирная линия) периодической квадратной решетки. Все решетки имеют одну и ту же максимальную глубину потенциала $V_{0}=12.5$ и значение фонового волнового числа $K=2 \pi$. Заметим, что с убыванием $\mu$ солитоны становятся у́же.

римановы суммы с $\Delta \theta \equiv 2 \pi / N$ :

$$
\begin{aligned}
\lim _{N \rightarrow \infty} V_{N}(x, y) & =\frac{V_{0}}{(2 \pi)^{2}} \lim _{\Delta \theta \rightarrow 0}\left|\sum_{n=0}^{2 \pi / \Delta \theta-1} e^{i K\left(x \cos \theta_{n}+y \sin \theta_{n}\right)} \frac{1}{\Delta \theta}\right|^{2}= \\
& =\frac{V_{0}}{(2 \pi)^{2}}\left|\int_{0}^{2 \pi} e^{i K(x \cos \theta+y \sin \theta)} d \theta\right|^{2}=V_{0} J_{0}^{2}(K r),
\end{aligned}
$$

где $r=\sqrt{x^{2}+y^{2}}$, а $J_{0}(r)$ - функция Бесселя нулевого порядка, интегральное представление которой [27] мы использовали. При возрастании $N$ для любого заданного радиуса угловое расстояние между решеточными максимумами (и минимумами) убывает, и предельная решетка Бесселя $(N=\infty)$ приобретает непрерывные кольцевые максимумы (рис. 11). Заметим, что солитоны Бесселя в последнее время были предметом исследований [28].

Из рис. $12(\mathrm{a})$ видно, что квазикристаллическая решетка 10-го порядка $(N=10)$ существенно отличается от квазикристаллической решетки Пенроуза $(N=5)$. С другой стороны, квазикристаллическая решетка 10-го порядка очень похожа на решетку Бесселя $\left(N=\infty\right.$, или $J_{0}^{2}(r)$ на рисунке). Поэтому солитонные профили, соответствующие квазикристаллической решетке 10-го порядка и решетке Бесселя, приблизительно одинаковы. Это проявляется особенно отчетливо в случае узких солитонов, поскольку различие между решетками заметно в области крыльев, а вблизи начала координат они почти тождественны. Однако для широких солитонов зависимость мощности от собственных значений существенно меняется. Более 

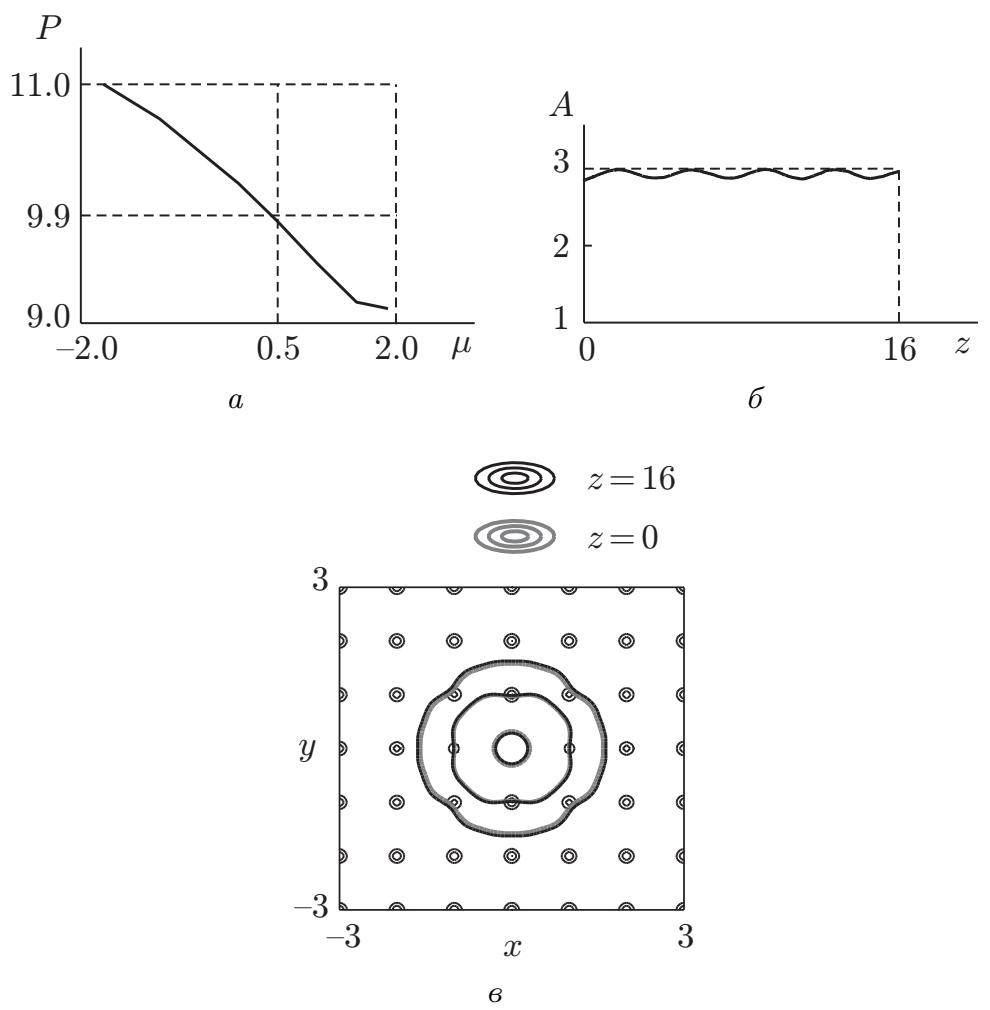

Рис. 8. Эволюция солитона, расположенного в вакансии потенциала (см. формулу (4) при $V_{0}=12.5$ и $K=2 \pi$, а также рис. 1 ), $\mu=0.5$. При таких значениях параметров наклон мощности (т.е. $d P / d \mu$ ) отрицателен (а). Пиковая амплитуда $A(z)=\max _{x, y}|u(x, y, z)|$ решения уравнения (1) как функция длины распространения. В качестве начальных условий взят солитон с однопроцентным шумом в амплитуде и фазе. В процессе распространения солитон остается очень устойчивым (б). Контуры начального солитона $(z=0)$ и почти неотличимого от него решения после прохождения шестнадцати дифракционных длин $(z=16)$ на фоне потенциала вакансии (в).

точно, при возрастании $N$ край щели убывает,

$$
\mu_{\max }\left[V_{N}\right] \stackrel{N \rightarrow \infty}{\rightarrow}+0
$$

а мощность на краю щели неограниченно возрастает (см. рис. 12б).

\section{9. ЗАКЛЮЧЕНИЕ}

Мы продемонстрировали существование устойчивых 2D-солитонов в самофокусирующейся среде с нерегулярными решеточными потенциалами, имеющими дефекты вакансий, дислокации и квазикристаллические структуры. На основе результатов расчетов можно сделать вывод, что солитоны Пенроуза аналогичны солитонам с 

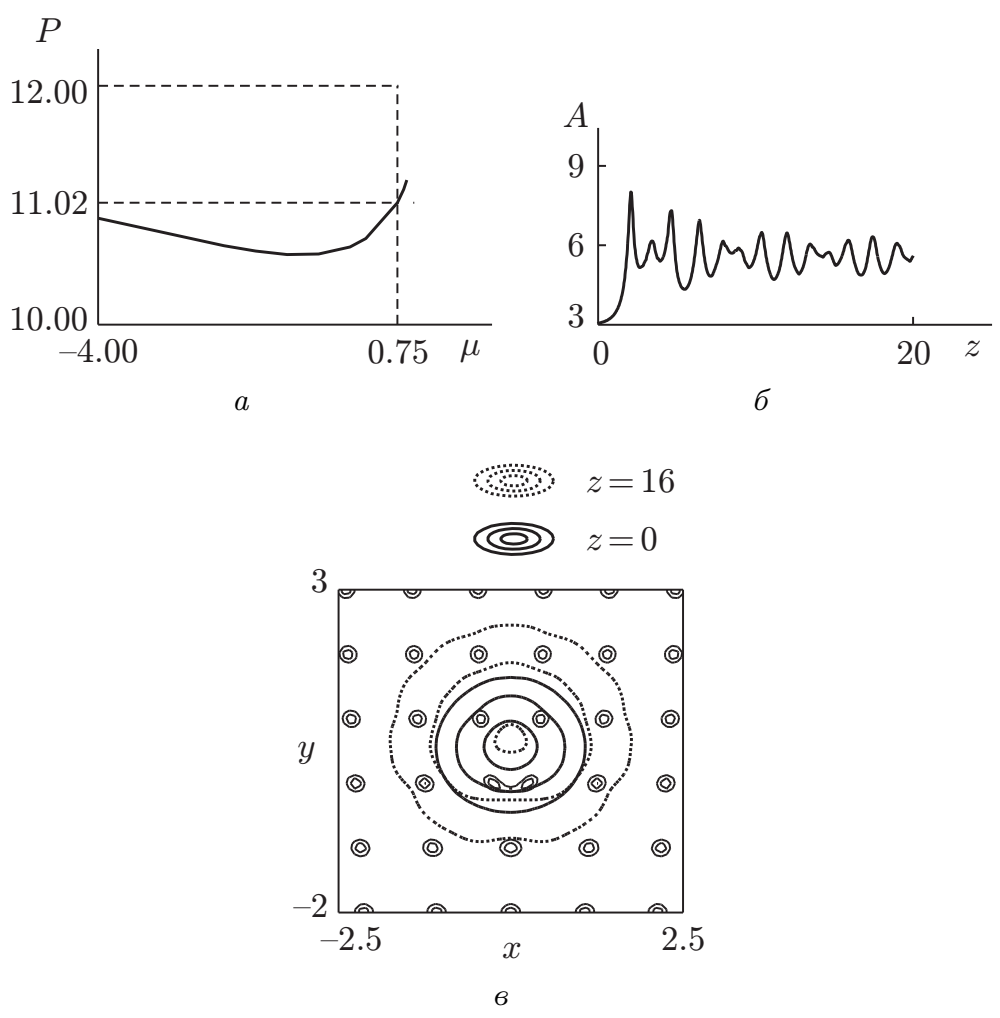

Рис. 9. То же, что на рис. 8 , для солитона на решетке с краевыми дислокациями (см. формулу (5) и рис. 2$), \mu=0.75$. При таких значениях параметров наклон мощности положителен (а). В процессе распространения решение претерпевает большие, но ограниченные фокусирующие-дефокусирующие колебания (б). Решение значительно сужается книзу, т.е. ширина на половине максимального значения величины $|u(x, y, z)|^{2}$ убывает приблизительно в два раза при изменении $z$ от начального значения $z=0$ (сплошная линия) к конечному значению $z=20$ (точки) (в).

центрами вблизи максимумов периодической решетки; с другой стороны, имеются существенные различия между солитонами с центрами вблизи вакансий и краевых дислокаций по сравнению с соответствующими им солитонами на периодической решетке.

\section{ПРИЛОЖЕНИЕ}

\section{Численный метод для вычисления солитонов}

Для решения уравнения (2) мы используем спектральный вычислительный метод с неподвижной точкой [18], суть которого объяснена ниже. Применяя преобразование Фурье к уравнению (2), добавляя и вычитая слагаемое $r \hat{u}$, где $r>0$ - константа, 

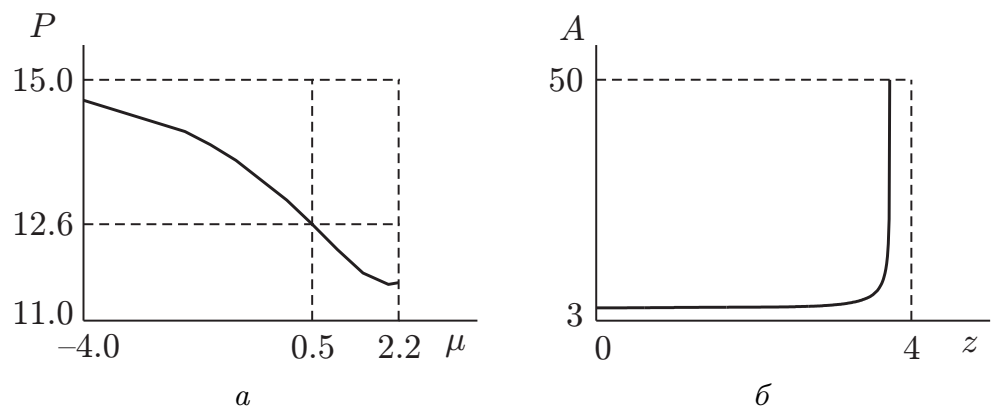

Рис. 10. То же, что на рис. 8а, 8б, для солитона на решетке Пенроуза (см. формулу (6) и рис. 4), $\mu=0.5$. При таких значениях параметров наклон мощности отрицателен (а). Решение претерпевает коллапс при $z \approx 3.7$, т.е. его пиковая амплитуда неограниченно возрастает, в то время как ширина убывает до нуля (б).
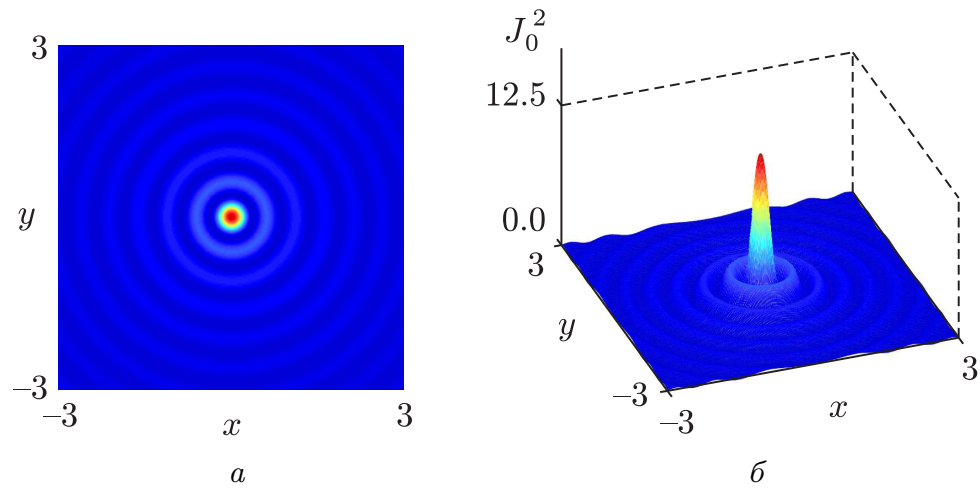

Рис. 11. Контурное (а) и трехмерное (б) изображения решетки Бесселя.

получаем

$$
\hat{f}(\nu)=\widehat{R}[\hat{f}] \equiv \frac{(r+\mu) \hat{f}+\mathcal{F}\left\{\left[|f|^{2}-V(x, y)\right] f\right\}}{r+|\nu|^{2}},
$$

где $\nu=\left(\nu_{x}, \nu_{y}\right)$ - переменные Фурье, $\mathcal{F}$ обозначает преобразование Фурье, а константа $r$ нужна для того, чтобы обойти особенность в знаменателе (мы используем значение $r=5)$. Введем новую полевую переменную $f(x, y)=\lambda w(x, y)$, где $\lambda \neq 0$ - константа, подлежащая определению. Метод итераций принимает вид $\widehat{w}_{m+1}=\lambda_{m}^{-1} \widehat{R}\left[\lambda_{m} \widehat{w}_{m}\right], m=0,1,2, \ldots$, где $\lambda_{m}$ удовлетворяет соответствующему алгебраическому условию

$$
\iint_{-\infty}^{+\infty}\left|\widehat{w}_{m}(\nu)\right|^{2} d \nu=\lambda_{m}^{-1} \iint_{-\infty}^{+\infty} \widehat{R}\left[\lambda_{m} \widehat{w}_{m}\right] \widehat{w}_{m}^{*}(\nu) d \nu .
$$

Было обнаружено, что данный метод предотвращает расходимость численной схемы. Таким образом, солитон получается в результате применения сходящейся ите- 

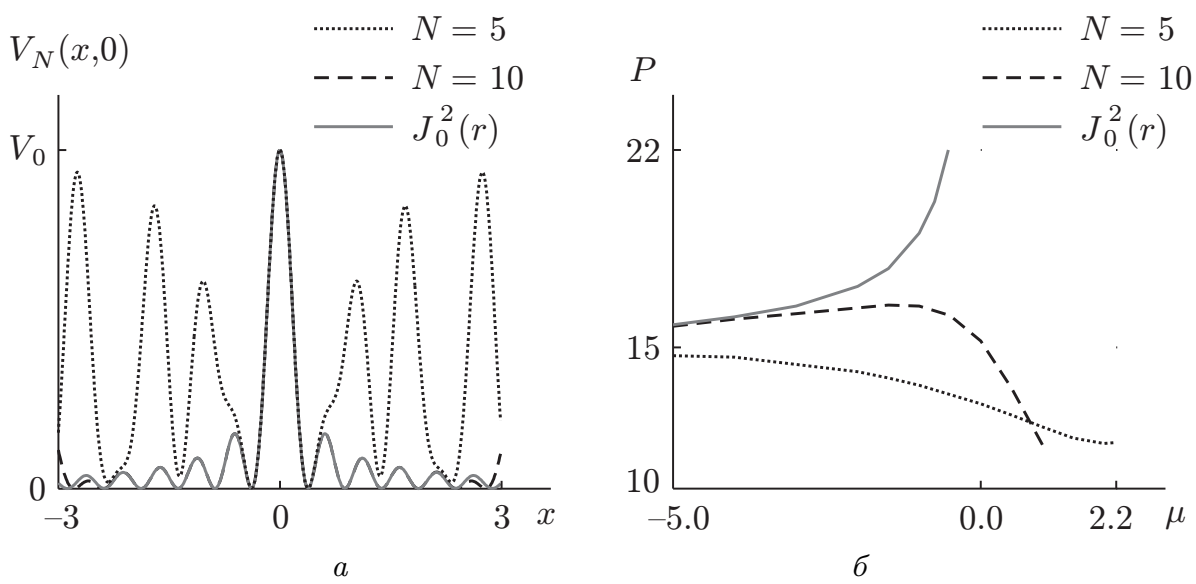

Рис. 12. Сечения $V_{N}(x, 0)$ потенциалов $V_{N}(x, y)$, заданных в $(7)$, при $V_{0}=12.5$ и $K=2 \pi$ для квазикристаллической решетки Пенроуза $(N=5$, пунктирная кривая), квазикристаллической решетки 10-го порядка $(N=10$, штриховая кривая, почти не отличающаяся от сплошной кривой) и решетки Бесселя $(N=\infty$, сплошная кривая) (а). Зависимость мощности от величины константы распространения для солитонов на трех соответствующих решетках (б).

рационной процедуры (см. также [29] по поводу альтернативной процедуры в случае, когда имеется хорошо определенная однородность). Начальная точка $w_{0}(x, y)$ обычно выбирается гауссовой. Итерации прекращаются, когда относительная погрешность $\delta=\left|\frac{\lambda_{m+1}}{\lambda_{m}}-1\right|$ достигает величины $10^{-10}$. Заметим, что сходимость является достаточно быстрой, но замедляется при расширении моды, т.е. когда $\mu$ приближается к краю (нелинейной) щели. Заметим также, что при подходящих предположениях относительно потенциала сходимость аналогичного метода была доказана в работе [30].

Благодарности. Данная работа выполнена при частичной финансовой поддержке US Air Force Office of Scientific Research (грант FA9550-06-1-0237) и NSF (грант DMS0505352).

\section{Список литературы}

[1] D. N. Christodoulides, F. Lederer, Y. Silberberg, Nature, 424 (2003), 817.

[2] A. A. Sukhorukov, Y.S. Kivshar, H.S. Eisenberg, Y. Silberberg, IEEE J. Quant. Electronics, 39 (2003), 31.

[3] N. K. Efremidis, J. Hudock, D. N. Christodoulides et al., Phys. Rev. Lett., 91 (2003), 213906.

[4] J. W. Fleischer, M. Segev, N. K. Efremidis, D. N. Christodoulides, Nature, 422 (2003), 147.

[5] D. Neshev, Y.S. Kivshar, H. Martin, Z. Chen, Opt. Lett., 29 (2004), 486.

[6] D. Shechtman, I. Blech, D. Gratias, J. W. Cahn, Phys. Rev. Lett., 53 (1984), 1951.

[7] M. Senechal, Quasicrystals and Geometry, Cambridge Univ. Press, Cambridge, 1995.

[8] M. P. Marder, Condensed Matter Physics, Wiley, New York, 2000. 
[9] F. Fedele, J. Yang, Z. Chen, Stud. Appl. Math., 115 (2005), 279.

[10] H. Buljan, G. Bartal, O. Cohen et al., Stud. Appl. Math., 115 (2005), 173.

[11] L. Guidoni, C. Triché, P. Verkerk, G. Grynberg, Phys. Rev. Lett., 79 (1997), 3363.

[12] E. Schonbrun, R. Piestun, Opt. Eng., 45 (2006), 028001.

[13] T. Pertsch, U. Peschel, F. Lederer et al., Opt. Lett., 29 (2004), 468.

[14] M. Qi, E. Lidorikis, P. T. Rakich et al., Nature, 429 (2004), 538.

[15] W. Cai, R. Piestun, Appl. Phys. Lett., 88 (2006), 111112.

[16] C. J. Pethick, H. Smith, Bose-Einstein Condensation in Dilute Gases, Cambridge Univ. Press, Cambridge, 2001.

[17] J. F. Nye, M. V. Berry, Proc. R. Soc. Lond. Ser. A, 336 (1974), 165.

[18] M. J. Ablowitz, Z. H. Musslimani, Opt. Lett., 30 (2005), 2140.

[19] M.S.P. Eastham, The Spectral Theory of Periodic Differential Equations, Texts Math., Scottish Acad. Press, Edinburgh-London, 1973.

[20] R. T. Bratfalean, A. C. Peacock, N. G. R. Broderick et al., Opt. Lett., 30 (2005), 424.

[21] R. Lifshitz, A. Arie, A. Bahabad, Phys. Rev. Lett., 95 (2005), 133901.

[22] W. Man, M. Megens, P. J. Steinhardt, P. M. Chaikin, Nature, 436 (2005), 993.

[23] L. Sanchez-Palencia, L. Santos, Phys. Rev. A, 72 (2005), 053607.

[24] B. Freedman, G. Bartal, M. Segev et al., Nature, 440 (2006), 1166.

[25] P. Xie, Z.-Q. Zhang, X. Zhang, Phys. Rev. E, 67 (2003), 026607.

[26] A. Della Villa, S. Enoch, G. Tayeb et al., Phys. Rev. Lett., 94 (2005), 183903.

[27] Справочник по спечиальным функииям с формулами, графиками и математическими таблицами, ред. М. Абрамовиц, И. Стиган, Наука, М., 1979.

[28] Y. V. Kartashov, V. A. Vysloukh, L. Torner, Phys. Rev. Lett., 93 (2004), 093904.

[29] Z. H. Musslimani, J. Yang, J. Opt. Soc. Amer. B, 21 (2004), 973.

[30] D. E. Pelinovsky, A. A. Sukhorukov, Y.S. Kivshar, Phys. Rev. E, 70 (2004), 036618. 